\title{
Severe hypothyroidism due to autoimmune thyroiditis in a child: a one-year follow-up
}

\author{
Francesco Vierucci, Graziano Cesaretti
}

Pediatric Endocrinology, Department of Pediatrics, "S. Chiara” University-Hospital, Pisa, Italy

Dear Editor,

Autoimmune thyroiditis is considered to be the most common autoimmune condition, with a female preponderance of $2: 1{ }^{1,2}$ While its overall prevalence peaks in adulthood, autoimmune thyroiditis may affect children and adolescents, particularly during early to mid-puberty. ${ }^{3}$ Hypothyroidism secondary to autoimmune thyroiditis may develop insidiously with slow and progressive physical changes that may remain unrecognized even to parents. ${ }^{4}$ Nevertheless, early recognition of symptoms and signs of hypothyroidism is important to prevent its negative effects on growth, metabolic function, and intellectual performance.

An eleven-year-old Caucasian boy was evaluated for poor linear growth, with normal weight gain starting at the age of six and never investigated previously. Physical examination showed short stature (height: -2.2 SDS; target height: 0.1 SDS) with overweight (body mass index: +1.7 SDS), bradycardia, sparse-brittle hair, dry and thickened skin with myxedema involving the extremities (Figure 1). The child appeared

Key words: Autoimmune thyroiditis, Childhood, Hypothyroidism, Myxedema

Address for correspondence:

Francesco Vierucci, M.D., Pediatric Endocrinology, Department of Pediatrics, "S. Chiara" University-Hospital, via Roma 67, 56126 Pisa, Italy, Tel.: +39 050 992101, E-mail: vieruf@hotmail.it

Received 16-01-2014, Accepted 19-03-2014 severely hypokinetic with apathy and mildly reduced intellectual performance (low-normal IQ score of 81, assessed by Wechsler Intelligence Scale for Children, third edition-WISC III). He also reported fatigue, constipation, sleepiness, impaired school performance, and cold intolerance.

Bone age was severely delayed (6 years as assessed by the Greulich and Pyle standard). Serum thyroid-stimulating hormone (TSH) levels were remarkably increased $(1,648 \mathrm{mIU} / \mathrm{L})$ with undetectable concentrations of thyroid hormones and elevated anti-microsomal and anti-thyroid peroxidase antibodies titers $(>2,040 \mathrm{IU} / \mathrm{mL}$ and $>1,020 \mathrm{IU} / \mathrm{mL}$, respectively). Thyroid ultrasound disclosed hypoechoic, inhomogeneous, and atrophic thyroid gland, confirming the diagnosis of severe hypothyroidism secondary to autoimmune thyroiditis.

Biochemical evaluation also revealed hypertransaminasemia (aspartate aminotransferase 151 $\mathrm{U} / \mathrm{L}$, alanine aminotransferase $117 \mathrm{U} / \mathrm{L}$ ), dyslipidemia (total cholesterol $360 \mathrm{mg} / \mathrm{dL}$, high-density lipoprotein cholesterol $29 \mathrm{mg} / \mathrm{dL}$, low-density lipoprotein cholesterol $294 \mathrm{mg} / \mathrm{dL}$, triglycerides $221 \mathrm{mg} / \mathrm{dL}$ ), and rhabdomyolysis (creatine kinase 6,883 U/L). Cerebral MRI showed a pituitary mass of $18 \times 12 \times 10$ $\mathrm{mm}$ extending into the suprasellar region, compatible with pituitary hyperplasia.

Low-dose levothyroxine administration was promptly started and progressively increased [0.5 $\mu \mathrm{g} / \mathrm{Kg} / \mathrm{die}$ (considering ideal weight according to height) for 2 weeks followed by $0.75 \mu \mathrm{g} / \mathrm{Kg} / \mathrm{die}$ for 1 month, $1 \mu \mathrm{g} / \mathrm{Kg} /$ die for 1 month and $1.5 \mu \mathrm{g} / \mathrm{Kg} /$ die for 


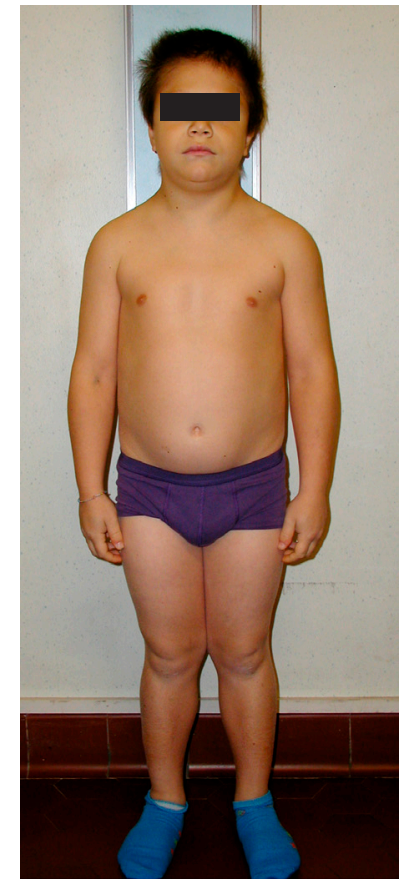

Figure 1. The patient at diagnosis with apathy, myxedema of the extremities, and sparse-brittle hair.

1 month, continuing with $2 \mu \mathrm{g} / \mathrm{Kg} /$ die afterwards]. Free thyroxine (fT4) and TSH levels normalized after 3.5 and 6 months of therapy, respectively. After twelve months of follow-up height improved to - 1.7 SDS. The child displayed normal hair, complete resolution of myxedema, normalization of biochemical alterations and intellectual performance (IQ score 92) (Figure 2). Finally, MRI showed the resolution of pituitary hyperplasia.

The case we describe herein illustrates that forms of severe juvenile hypothyroidism are still relevant today. It seems unbelievable that nobody had recognized the progressive onset of severe hypothyroidism before the child developed severe multi-organ complications. Moreover, this case demonstrates that levothyroxine administration significantly improves the changes due to long-lasting hypothyroidism acquired during childhood.

A careful monitoring of growth is essential to avoid late diagnosis of diseases with possible longterm sequelae, such as acquired hypothyroidism. A child with poor linear growth should always be adequately investigated. Finally, the detection of a child with impaired linear growth associated with normal

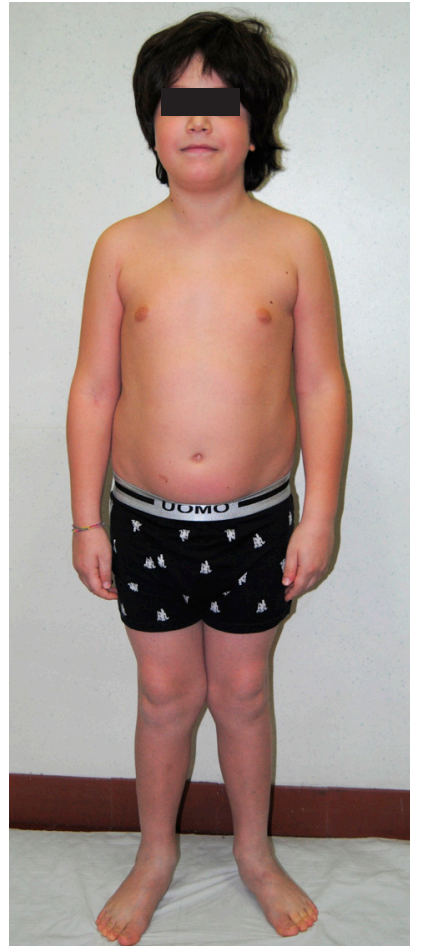

Figure 2. The patient after twelve months of therapy with levothyroxine. The resolution of myxedema and the normalization of hair growth were noticeable in association with the disappearance of apathy.

weight gain should always arouse the suspicion of hypothyroidism. ${ }^{4}$

\section{DISCLOSURE STATEMENT:}

The authors have nothing to disclose.

\section{PATIENT CONSENT}

Obtained from the parents.

\section{REFERENCES}

1. Saravanan P, Dayan CM, 2001 Thyroid autoantibodies. Endocrinol Metab Clin North Am 30: 315-337.

2. Lafranchi S, 1992 Thyroiditis and acquired hypothyroidism. Pediatr Ann 21: 29-39.

3. Cappa M, Bizzarri C, Crea F, 2011 Autoimmune thyroid diseases in children. J Thyroid Res 2011: 675703.

4. Büyükgebiz A 2007 Newborn screening, hypothyroidism in infants, children and adolescents. In: Krassas GE, Rivkees SA, Kiess W (eds), Diseases of the thyroid in childhood and adolescence. Pediatr Adolesc Med Basel, Karger; pp, 128-141. 